- Careful patient assessment is the most important part of treatment

- The intra-oral examination is conducted after the extra-oral assessment

- The degree of occlusal discrepancy influences the treatment options

- The dental health and patient motivation determine if appliance therapy can be used

\title{
Orthodontics. Part 3: Patient assessment and examination II
}

\author{
D. Roberts-Harry ${ }^{1}$ and J. Sandy ${ }^{2}$
}

ORTHODONTICS
1. Who needs
orthodontics?
2. Patient assessment and
examination I
3. Patient assessment and
examination II
4. Treatment planning
5. Appliance choices
6. Risks in orthodontic
treatment
7. Fact and fantasy in
orthodontics
8. Extractions in
orthodontics
9. Anchorage control and
distal movement
10. Impacted teeth
11. Orthodontic tooth
movement
12. Combined orthodontic
treatment

$1^{*}$ Consultant Orthodontist, Orthodontic Department, Leeds Dental Institute, Clarendon Way, Leeds LS2 9LU; ${ }^{2}$ Professor in Orthodontics, Division of Child Dental Health, University of Bristol Dental School, Lower Maudlin Street, Bristol BS1 2LY ${ }^{*}$ Correspondence to: D. Roberts-Harry E-mail: robertsharry@btinternet.com

\section{Refereed Paper}

doi:10.1038/sj.bdj.4810724

( $)$ British Dental Journal 2003; 195 :

563-565

The intra-oral assessment examines the oral health, individual tooth positions and inter-occlusal relationships. When this has been completed in conjunction with the extra-oral examination, a treatment plan can then be formulated.

\section{INTRA-ORAL EXAMINATION}

There are various systems available to assess this aspect but the following sequence is both practical and thorough:

- Dental health

- Lower arch

- Upper arch

- Teeth in occlusion

- Radiographs

\section{Dental health}

Even individuals with severe malocclusions should not have active orthodontic treatment in the presence of dental disease. Orthodontic appliances accumulate plaque and if the patient has a poor diet and tooth brushing then irreversible damage can result as demonstrated in Figure 1. Although the patient has straight teeth there is considerable decalcification and it could be argued is worse off as a consequence of treatment. Clearly this could have serious medicolegal complications, particularly if the clinician fails to write in the notes that appropriate dental health advice has been given

Decalcification around orthodontic appliances is a recognised hazard and will occur in the presence of poor oral hygiene and a cariogenic diet. Not only will decalcification occur around the brackets but tooth movement in the presence of active gingivitis or periodontal disease will accelerate any bone loss. Attempting to move teeth in the presence of active dental disease can have disastrous consequences and must be avoided.

Therefore, treatment for patients with ques- tionable dental health should be confined to extractions and spontaneous alignment of the teeth only. Figure 2 illustrates a case where there is an obvious need for orthodontic treatment but this was precluded by the patient's extremely poor oral hygiene.

Minor apical root resorption is a common consequence of orthodontic tooth movement. However, this resorption can occasionally be severe. Tooth movement in the presence of apical pathology is known to accelerate resorption and should be dealt with prior to commencing treatment.

\section{Lower arch}

The lower arch should be examined and planned in the first instance. Whatever treatment is carried out in the lower arch often determines the treatment to be carried out in the upper. Examine the teeth for any tipping, rotations and crowding. Teeth which are tipped mesially are much more amenable to treatment, both with removable and fixed appliances than teeth which are distally tipped. They also respond much better to extractions and spontaneous alignment than other teeth. The presence or absence of rotations is important because rotated teeth are most easily treated with fixed appliances. The more crowded the teeth are the more likely it is that extractions will be needed in order to correct the malocclusion. A method of assessing crowding is given in Figure 3. Firstly, measure the size of the teeth and add these together (length A). Then measure from the mid-line to the distal of the canine with a pair of dividers. Measure from the distal of the canine to the mesial of the first permanent 

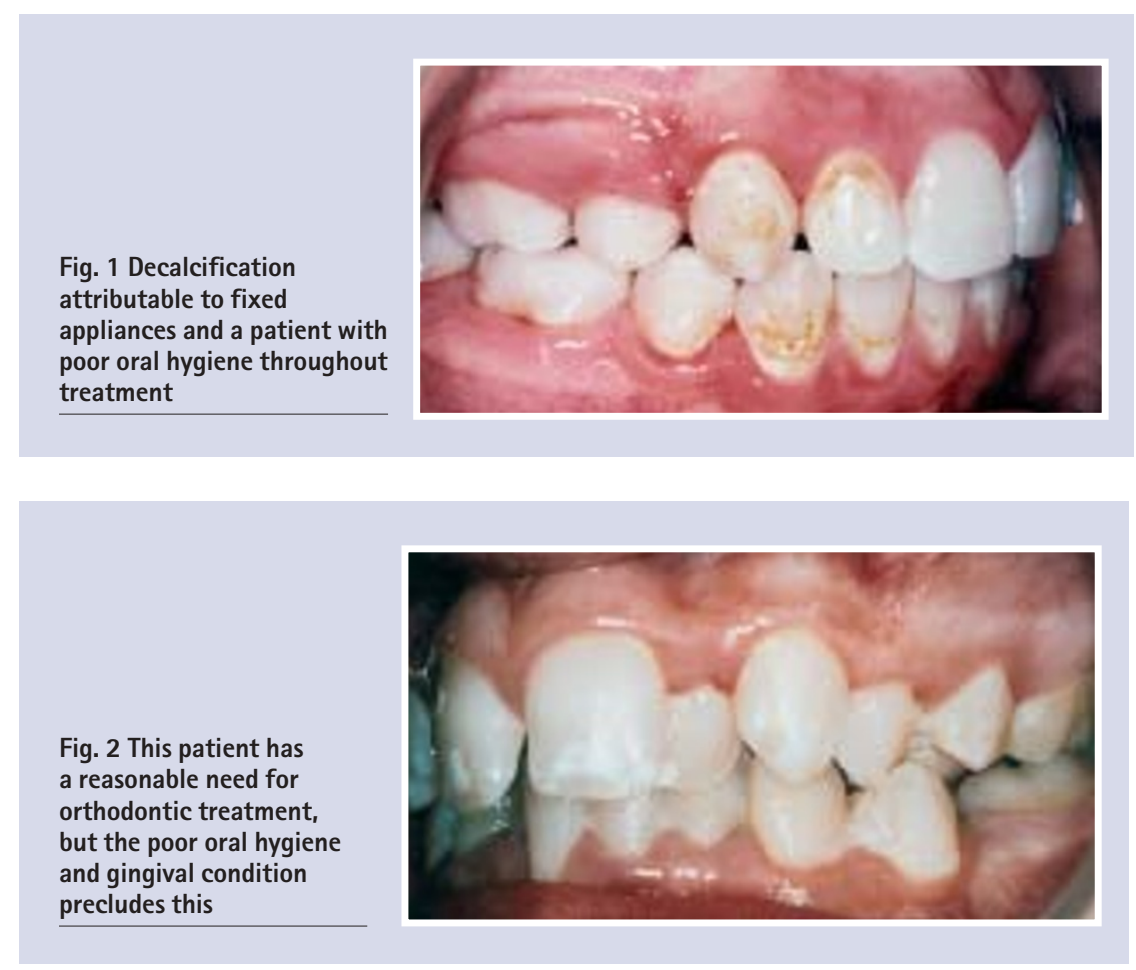

molar. Add these together to give you the approximate arch length (length B). Subtract B from A to give you the degree of crowding. This must be repeated for both sides of the arch.

The degree of crowing influences the need for extractions. Although one should not be dogmatic and several other factors influence the planning of extractions, as a general rule the greater the crowding the more likely extractions are necessary. Table 1 gives an outline of the relation between degree of crowding and need for extractions.

\begin{tabular}{lc}
$\begin{array}{l}\text { Table } 1 \text { Relationship between crowding and } \\
\text { extractions }\end{array}$ \\
\hline Degree of crowding & Need for extractions \\
\hline$<5 \mathrm{~mm}$ & No \\
\hline $5-10 \mathrm{~mm}$ & Possibly \\
$>10 \mathrm{~mm}$ & Yes
\end{tabular}

\section{Upper arch}

This is examined in a similar way to the lower arch. Additional points to note in the mixed dentition are the presence of a mid-line diastema and the position of the upper canines.

A mid-line diastema is commonly seen in the mixed dentition. The aetiological factors to be considered are:

- Normal (physiological) development

- Fraenum

- Small teeth

- Missing teeth

- Midline supernumerary

Physiologic spacing usually disappears as the occlusion matures, especially when the upper permanent canines erupt and no treatment apart from observation is needed. Fraenectomies are rarely indicated and generally do not need to be removed unless the fraenum is particularly large and fleshy.

The upper permanent canine should be palpable in the buccal sulcus by 10 years of age. If not, and the deciduous canine is firm, parallax radiography should be undertaken to determine where the permanent tooth is. If the tooth is palatally positioned then the deciduous canines on both sides should be removed. This will help guide the permanent tooth into a more favourable path of eruption and prevent any centre line shift caused by a unilateral deciduous extraction. It is essential that this palpation be carried out on all patients in this age group. Very often impacting canines are missed and the patient not referred for treatment until 15 or 16 years of age. Not only is this negligent, but the patient may then need to undergo a lengthy course of treatment at a socially difficult time.

\section{Teeth in occlusion}

The overjet and overbite should be measured and the incisor classification assessed. The British Standards Institute (BS EN21942 Part 1 (1992) Glossary of Dental terms) defines the incisor classification as follows:
Fig. 3 Assessment of crowding. The widths of all the teeth anterior to the molars are measured and subtracted from the sum of two measurements (mesial of the lower incisor to the distal of the lower canine, plus distal of lower canine to the mesial of the first molar) to give the degree of crowding

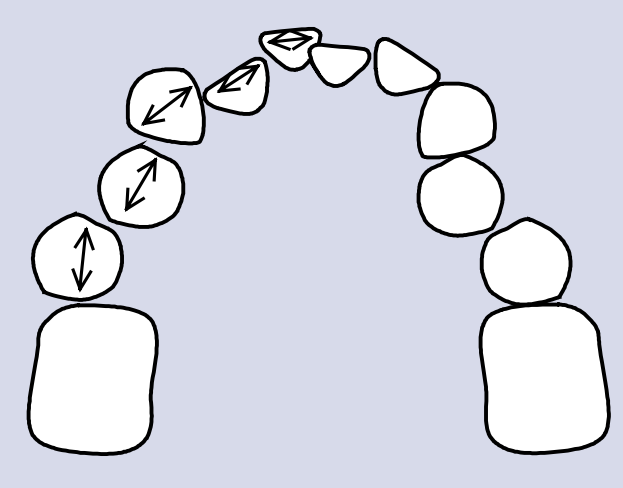

$1+2+3+4+5=\mathrm{A}$

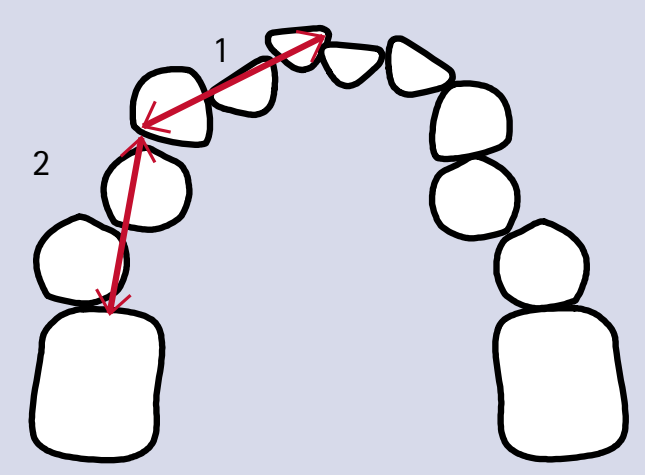

$1+2=B$ 


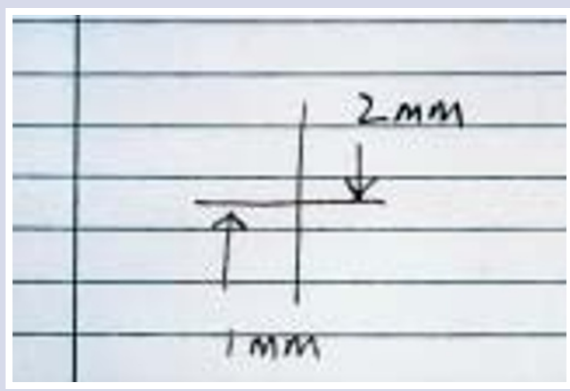

Fig. 5 Method for recording deviations in the centre line where the lower is to the right by $1 \mathrm{~mm}$ and the upper to the left by $2 \mathrm{~mm}$

- Class I. The lower incisor edges occlude with or lie immediately below the cingulum plateau (middle part of) the upper central incisors.

- Class II. The lower incisor edges lie posterior to the cingulum plateau of the upper central incisors. There are two divisions:

Division 1 - there is an increase in the overjet and the upper central incisors are usually proclined.

Division 2 - the upper central incisors are retroclined. The overjet is usually minimal but may be increased.

- Class III. The lower incisor edges lie anterior to the cingulum plateau of the upper central incisors. The overjet is reduced or reversed.
The centre line should be measured by placing a ruler down the patient's facial mid-line and measuring how far away from this the centre lines deviate (Fig. 4). This can then be marked in the notes as shown in Figure 5.

The buccal occlusion is assessed next, particularly the molar relationship. This is important because when assessing the treatment, it has to be decided whether the buccal occlusion is to be accepted or whether it should be corrected as part of the treatment plan. The canine and molar relationships should be recorded as class I, II or III

Finally, the presence of any anterior or posterior cross-bites should be assessed and if there is a cross-bite, the clinician should check to see whether there is any mandibular displacement associated with it. This is important because any displacement will mask the position of the teeth and give a misleading indication of the inter-occlusal relationships. Figure 6 shows a child who has an apparently severe class III incisor relationship. However, he can get his teeth into an edge-to-edge relationship and in this position the occlusion does not appear to be so severe. The amount of proclination of the upper incisors needed to correct the incisor relationship was quite mild and easily accomplished using a removable appliance (Fig. 7 and 8).

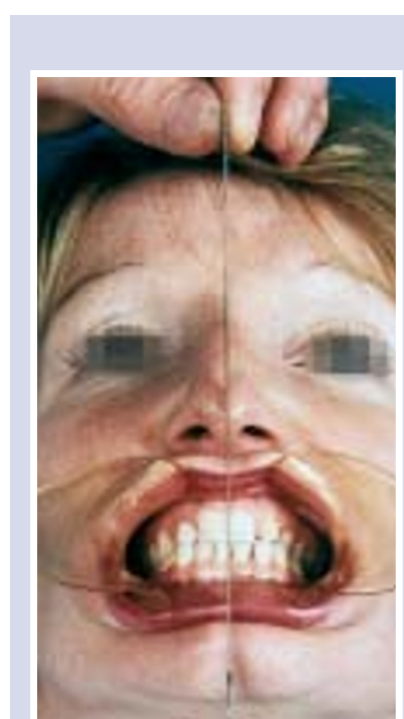

Fig. 4 Measurement of centre line deviation using a ruler placed in the patient's mid line

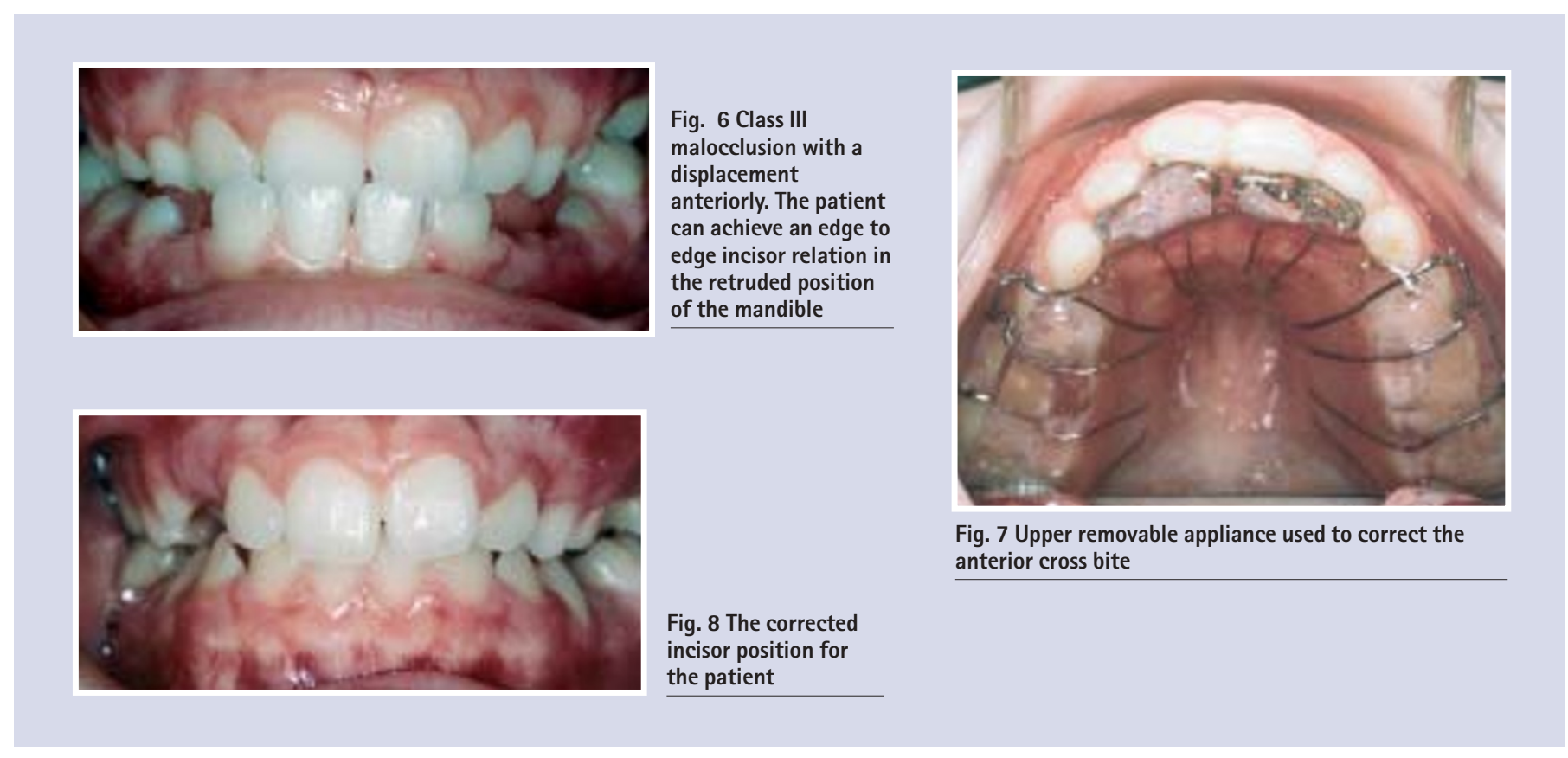

